\title{
SPATIALLY-RESOLVED 3 $\omega$ THERMAL FLOW SENSING FOR MICROFLUIDICS AND BIOLOGY
}

\author{
Claude Meffan ${ }^{1}$, Nicolas Cheradame ${ }^{1}$, Mathieu Sellier ${ }^{1}$, Emilio Calius $^{2}$ and Volker Nock ${ }^{1}$ \\ ${ }^{1}$ University of Canterbury, Christchurch, NEW ZEALAND and \\ ${ }^{2}$ Callaghan Innovation Ltd, Wellington, NEW ZEALAND
}

\begin{abstract}
This paper reports an alternating current $(\mathrm{AC})$ thermal flow sensor, based on the $3 \omega$ method, capable of measuring fluid flow in stacked microfluidic channels and through separating membranes. The measurement concept is tested in a triple-layer polydimethylsiloxane (PDMS) device containing two parallel channels separated by a membrane. A $3 \omega$ element integrated into the bottom channel was used to determine the flow direction and magnitude in both channels. Our results show that the phase of the temperature wave is linked not only to fluid velocity, but the physical dimensions of the channel, thus providing a novel non-contact tool to probe fluid flows.
\end{abstract}

\section{KEYWORDS}

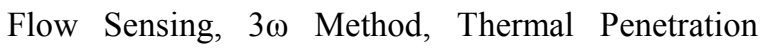
Depth, Polydimethylsiloxane Microfluidics.

\section{INTRODUCTION}

Standard fluid velocity sensors use a time-of-flight measurement concept between separate heater and thermometers elements. In contrast, the $3 \omega$ method uses a single resistive element as both heater and thermometer [1,2]. This method has historically been used to measure the thermal conductivity of solids and liquids [3]. It has proven particularly useful for the characterization of thermal properties of nanoparticle composites and fragile thin-films [4], which are otherwise difficult to characterize.

More recently, single $3 \omega$ alternating current (AC) resistance thermometers have been adapted for used as flow sensors in the macro- and microscale [5,6], and as biological assay tools to characterize multiscale thermal properties related to biomedical applications [7]. Among others, this has included the demonstration of the $3 \omega$ method as a tool to thermally detect disease at single cell level and to track disease progression in cancer [8].

One of the most important heat transfer concepts in the $3 \omega$ method is that of the thermal penetration depth. Due to the periodic nature of the heating, the heating frequency can be used to tune the thermal penetration depth [9]. This penetration depth corresponds to the distance that the amplitude envelope of the oscillating thermal wave penetrates above or below the heater element. More precisely, the lower the heating frequency, the longer the penetration depth, and vice versa, which enables the interrogation of thermal properties at various distances from the heating element.

In the present work we investigate the use of this concept for long-range characterization of fluid flow in direct contact and through solid membranes. For this, a $3 \omega$ element was integrated into a triple-layer polydimethylsiloxane (PDMS) microfluidic channel, and the concept of finite thermal penetration depth used to probe fluid flow vertically above the heater element. As such, thermal penetration depth of an AC thermal flow sensors was, for the first time, used as functional aspect to probe flow in stacked channels and through solid membranes at a distance.

\section{EXPERIMENTAL Device fabrication}

The study platform consisted of two $100 \times 100 \mu \mathrm{m} \times$ $35 \mathrm{~mm}(\mathrm{H} \times \mathrm{W} \times \mathrm{L})$ vertically-stacked PDMS microfluidic channels, each with their own inlet and outlet. A $20 \mu \mathrm{m}$ PDMS membrane separated the two independent channels and a $3 \omega$ resistance thermometer was aligned into the bottom channel, as shown in Fig. 1. Standard PDMS (10:1 w/w Sylgard 184, Dow Corning) soft-lithography and replica molding were used to fabricate the top channel [10]. The bottom channel, together with the $20 \mu \mathrm{m}$ membrane, were fabricated monolithically using PDMS exclusion molding [11]. Top and bottom channels were manually aligned and bonded using oxygen plasma (Tergeo, PIE Scientific) prior to peeling off from the bottom mould. This yielded two vertically stacked parallel channels, as indicated in the inset in Fig. 1.

A $3 \omega$ resistance thermometer (RT) element with four electrical contact pads was separately microfabricated on a soda-lime glass slide using photolithography and thermal evaporation of $200 \mathrm{~nm}$ thick gold [6]. This substrate with the RT on it was then manually aligned with the PDMS channels and again bonded using oxygen plasma. Prior to use, bonding and membrane continuity were tested by

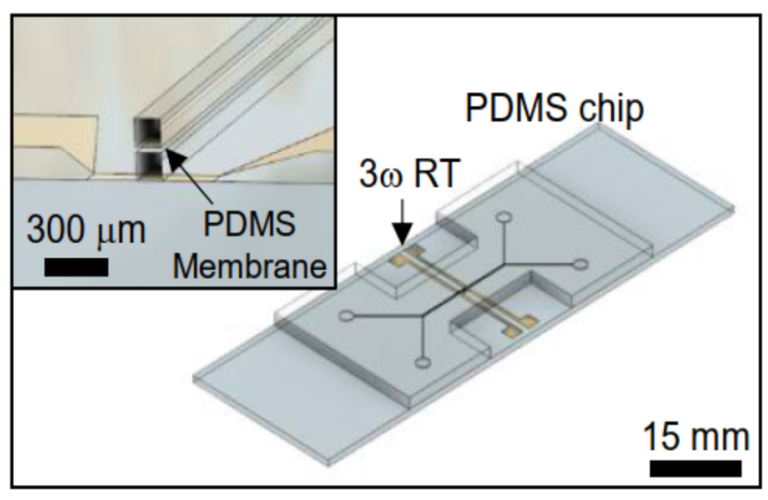

Figure 1: Schematic of the prototype microfluidic device, including the 4-point gold resistance thermometer (RT), used to demonstrate spatiallyresolved $3 \omega$ flow sensing. Top and bottom channels have individual inlets and outlets, and are separated from each other by the PDMS membrane. Inset: Crosssection of the device showing the vertical channel stacking, separating membrane and $R T$ integrated at the base of the bottom channel. 

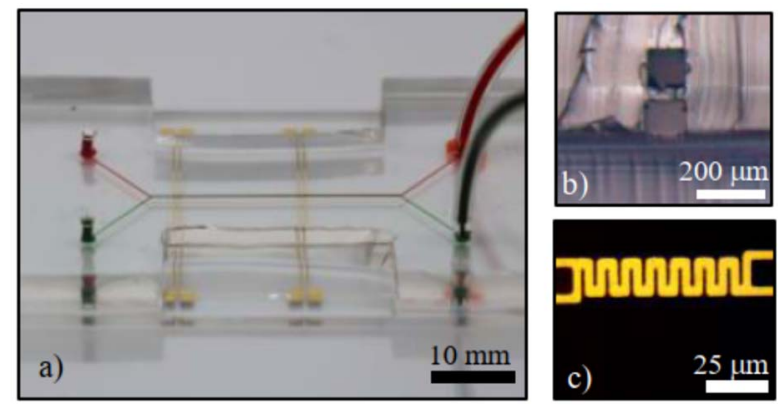

Figure 2: Vertically-stacked microfluidic device. (a) Photograph of the chip with two integrated $3 \omega$ sensing elements. Red and green food coloring was used to confirm channel and membrane integrity. (b) Cross-sectional optical micrograph of the chip showing channel alignment and membrane thickness. (c) Optical micrograph of the serpentine gold $R T$, integrated into the bottom channel and used to probe flow in both channels.

flowing dye-colored deionized (DI) water through the device and checking using optical microscopy.

\section{Flow actuation}

Calibrated DI-water flow in the channels was generated via a syringe pump (PHD2000, Harvard) and external flow sensors (MFS3, Elveflow) connected to the chip via PTFE tubing (IDEX HS). Flow was applied to the bottom channel, top channel, or both channels simultaneously, with stationary fluid acting as control. Experiments were performed at room temperature in a temperature-controlled room with the DI water equilibrated to room temperature.

\section{Thermal sensing}

The onboard signal-generator of a lock-in amplifier, controlled by LabOne software (MFLI, Zurich Instruments), was used to pass an AC heating current through the $3 \omega$ RT. Simultaneously, the same lock-in amplifier was used to record the resulting 3rd harmonic voltage, which was then transformed into a temperature response [2,6]. A frequency sweep from $0.2 \mathrm{~Hz}$ to $500 \mathrm{~Hz}$ was used to probe $4.8 \mu \mathrm{m}$ to $240 \mu \mathrm{m}$ into the device, covering the full depth of both channels and the separating membrane. The harmonic frequency response of the $3 \omega$ RT was then related to flow at height via the thermal penetration depth $\lambda_{\text {thermal }}$ as follows:

$$
\lambda_{\text {thermal }}=\sqrt{\frac{D_{\text {fluid }}}{\omega_{H}}}
$$

where $D_{\text {fluid }}$ is the thermal diffusivity of the fluid and the heating frequency $\omega_{H}=2 \omega_{\text {electrical [6]. This thermal }}$ wavelength was calculated, in first approximation, assuming homogenous material throughout. However, it should be noted that thermal waves will travel further in PDMS than in water.

\section{RESULTS AND DISCUSSION Microfluidic device}

Microfabrication, based on PDMS replica-moulding, metal deposition and photolithography, was chosen to fabricate the experimental platform, as this technology enable the reproduction of channel dimensions which could be covered by the finite thermal penetration depth. Due to the relationship between frequency and penetration depth, the maximum distance fluid flow above the heater element can be investigated is limited to a few hundred micrometers. This limitation mainly arises from the fact that otherwise frequency scan times would become prohibitively long.

A photograph of the fabricated microfluidic device is shown in Fig. 2, including a cross-section of the two vertically-stacked PDMS channels and a close-up of the integrated $3 \omega$ gold RT. The overall height of the fluidic structure above the heater was $220 \mu \mathrm{m}$, which includes the top and bottom channels, as well as the separating PDMS membrane. The thickness of $20 \mu \mathrm{m}$ for the latter was chosen to simplify fabrication, but could be reduced or increased in the future. Optical inspection demonstrated that manual alignment was sufficient to yield parallel channels over their full length of $35 \mathrm{~mm}$. As shown in Fig. 2(c), a serpentine RT design was chosen to increase the element resistance to $5.6 \Omega$ and thus the $3 \omega$ signal strength. Initial tests showed that applying a heating power of $16 \mu \mathrm{W}$ to the RT using the lock-in amplifier generated an $\mathrm{AC}$ temperature rise $<10^{\circ} \mathrm{K}$.

\section{Single channel flow}

Prior to experiments with fluid flow, the thermal response of the platform was measured for stationary DI water in both the top and bottom channels. This measurement was used as control in the following flow experiments. Figure 3 shows the temperature phase shift changing relative to the control sweep, indicated by the black vertical line, and respective flow arrangement. For the first case, shown in Fig. 3(a), the fluid in the top channel was left stationary, while the bottom channel was actuated to 2, 3 and $5 \mu \mathrm{l} / \mathrm{min}$ of pressure driven flow. The direction of flow was from left to right as indicated by the parabolas in this and following figures. Scanning the thermal penetration depth through the vertical stack resulted in a strong dependence of the phase shift on the applied flowrate in the bottom channel. At distances from the RT corresponding to the top of the bottom channel (100 $\mu \mathrm{m}$ and above), this phase shift can be observed to begin to decay through the stationary fluid in the top channel back towards the control case measurement.

In the second case, shown in Fig. 3(b), the flow conditions were reversed, such that the fluid in the bottom channel was left stationary, while the top channel was actuated to 2, 3 and $5 \mu \mathrm{l} / \mathrm{min}$ of pressure driven flow. In this case the phase shift response of the RT remained closely aligned with the stationary control up to the vertical location of the separating membrane at $100 \mu \mathrm{m}$. Beyond this level, a clear dependence of the phase shift on the applied flowrate in the top channel could be observed. The decay back to control in this case is not visible as the measurements only extended vertically to $240 \mu \mathrm{m}$.

In general, we found that in our measurements the phase responded to fluid velocity to a greater extent than the temperature magnitude [5]. As summarized in Fig. 4, the phase scaled linearly with the flow-speed in the tested 


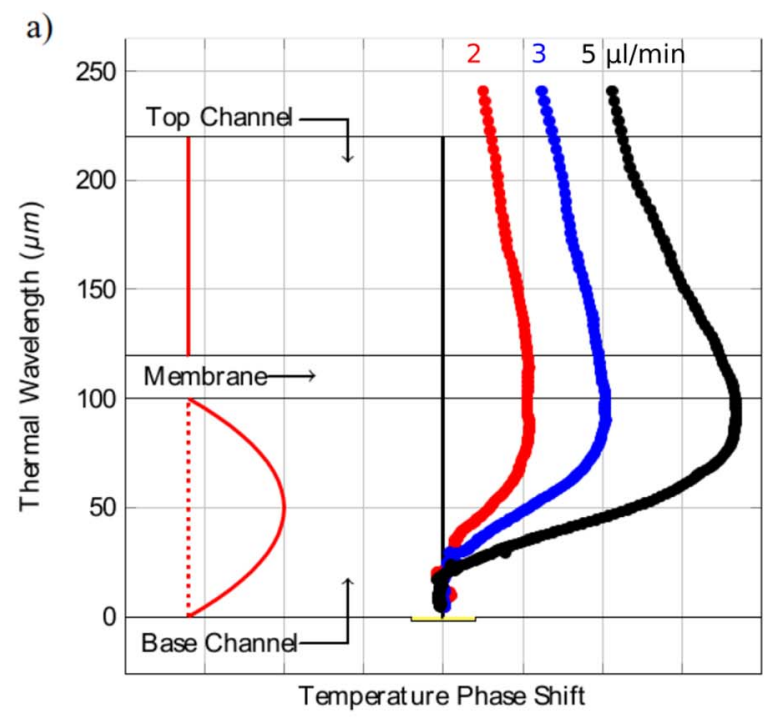

b)

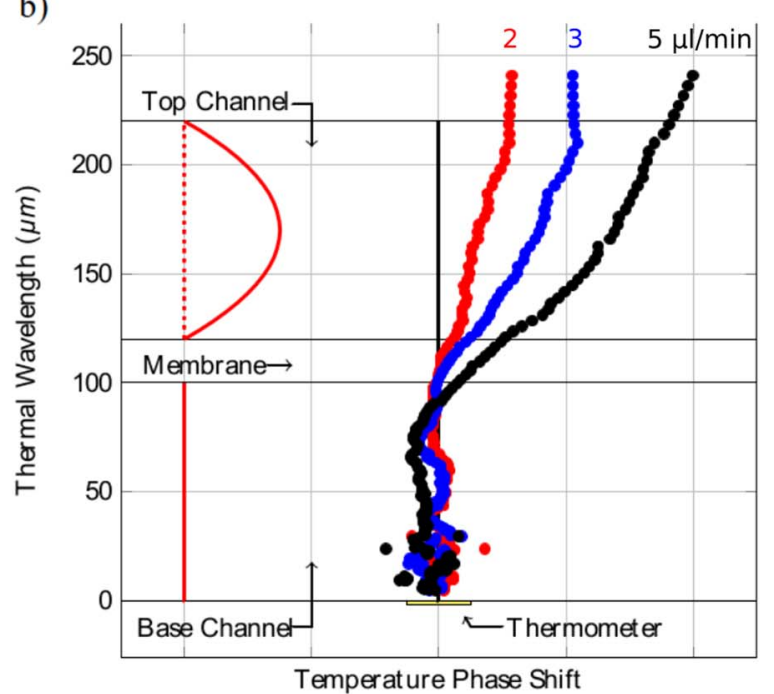

Figure 3: Flow-dependent response of the $3 \omega$ sensor. (a) Fluid flow is actuated in the bottom channel only. The 3rd harmonic response varies from the control measurement (black line) in proportion to the fluid velocity, and in line with the physical dimensions of the channel. (b) Fluid flow is actuated in the top channel only. The sensor response is consistent with the control until $1.1 \mathrm{~Hz}(100 \mu \mathrm{m}$ thermal penetration depth). This aligns with the bottom of the top channel.

range from $2 \mu \mathrm{l} / \mathrm{min}$ to $5 \mu \mathrm{l} / \mathrm{min}$ for both cases of top and bottom only flow. For bottom channel only flow the gradient of phase shift versus flow rate at the middle of the bottom channel ( $50 \mu \mathrm{m}$ depth) was observably steeper than at the middle of the top channel $(170 \mu \mathrm{m}$ depth) for top channel only flow. This does indicate that there is a limit to how far vertically above the RT one can reliably probe using this technique. In general, the low thermal frequencies used to probe long distances can take an unpractically long time to reach thermal equilibrium, and thus require static conditions or developed flow. However, corner frequencies in the response aligned well with the physical dimensions of the channel and, as Fig. 3(b) demonstrates, the sensor responds uniquely to fluid flow in the top channel for the geometry and scales of the setup.

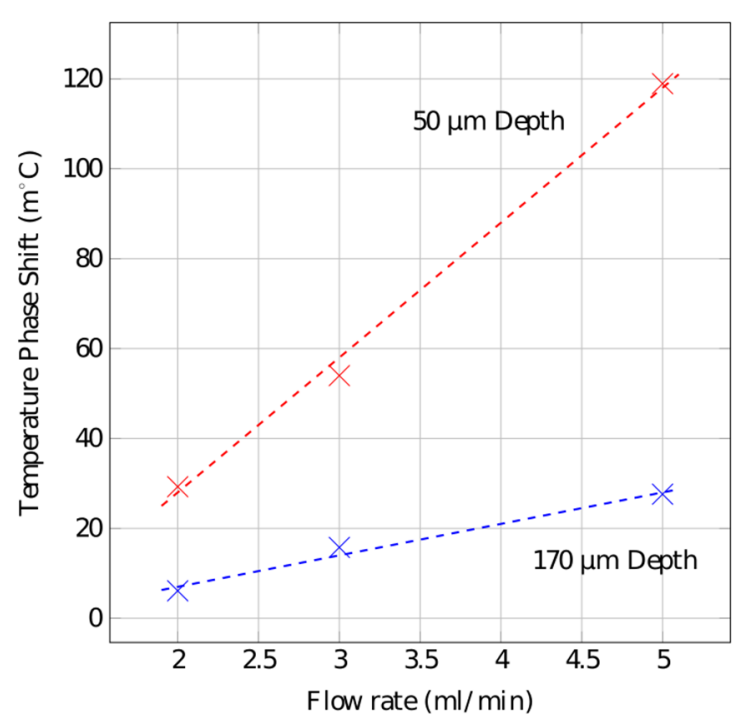

Figure 4: Plot of temperature phase shift versus flow rate. The red line shows the linear relationship between temperature phase shift and flow rate at a thermal penetration depth of $50 \mu \mathrm{m}$ (mid-channel, bottom channel) for bottom channel flow only. In comparison, the gradient of the same relationship at $170 \mu \mathrm{m}$ (mid-channel, top channel) for flow in the top channel only is significantly shallower.

\section{Simultaneous, dual-channel flow}

Interestingly, in the case of bi- or unidirectional simultaneous flow in both channels, the sensor response becomes much more complex. Figure 5 shows an example of the comparison of the temperature phase shift plots when flow is present in both channels at the same time. For this example, the flow in the top and bottom channels was actuated to the same flow rate of $4 \mu \mathrm{l} / \mathrm{min}$ in both channels. In the unidirectional case, flow in both channels was in the same direction and of the same flow rate as indicated by the red and blue parabolas. In contrast, in the bidirectional case, the flow in the top channel was reversed into the opposite direction to that in the bottom channels, as indicated by the red parabolas, while maintaining the same flow rate in each. The temperature phase shift response for both cases was again plotted in relation to the stationary case, indicated by the black line in Fig. 5 .

As can be observed, in the bidirectional case the shape of the temperature phase shift in the top channel was noticeably shifted in relation to the unidirectional case. This shift was in direction of the reversed flow in the top channel, indicating that the flow did influence the thermal transport. However, the magnitude of this phase in the bidirectional case was not as large as might be expected for opposing flows of the same flow rate. More work is thus needed to fully understand the interaction of thermal transport in this case and modeling is currently underway to better understand the observed behavior.

Nonetheless, our results validate the concept of using the frequency response to make spatially-resolved flow measurements at a distance and in compartmentalized setups through solid dividers, such as PDMS membranes. Future work will test the applicability of the principle to, in 


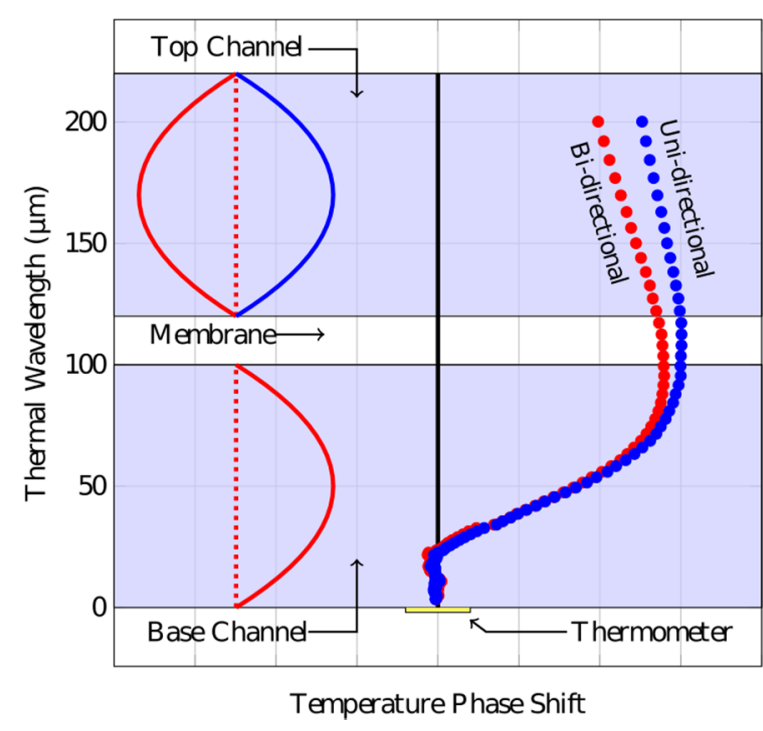

Figure 5: Flow-dependent response of the $3 \omega$ sensor for simultaneous flow of $4 \mu \mathrm{l} / \mathrm{min}$ in both channels, either bi- (red dotted line) or uni-directional (blue dotted line). While the bi-directional temperature phase shift is reduced compared to the uni-directional case, the difference is not as large as may be expected for equal flow rates in both directions.

particular, biological systems, where an internal flow is separated from an external static fluid environment by a membrane. Examples of such are growing pollen tubes [12], root tips and hairs [13], and fungal hyphae [14], all which exhibit difficult to measure fluid absorption and internalized mass transport. By allowing microorganisms with internal fluid-flow to grow across the sensor, thermal penetration depth could then be used to probe flow internal to the organisms in a non-contact manner.

\section{CONCLUSIONS}

We have demonstrated the first use of an AC thermal flow sensor, based on the $3 \omega$ method, for the measurement of fluid flow in stacked microfluidic channels and through separating membranes. A triple-layer PDMS microfluidic device, containing two parallel channels separated by membrane and a $3 \omega$ element integrated into the bottom channel, was used to determine the flow direction and magnitude of fluid flow in both channels. The phase of the temperature wave was found to be linked not only to fluid velocity, but also the physical dimensions of the channel. This illustrates that thermal penetration depth can be used as a novel non-contact technique to probe fluid flow and mass transport in complex geometrical configurations.

\section{ACKNOWLEDGEMENTS}

The authors would like to thank Gary Turner and Helen Devereux for technical assistance. Financial support was provided by Callaghan Innovation (C08X1211) and the MacDiarmid Institute for Advanced Materials and Nanotechnology.

\section{REFERENCES}

[1] N.O. Birge, and S.R. Nagel, "Wide frequency specific heat spectrometer.” Rev. Sci. Instrum., vol. 58, no. 8, pp. 1464-1470, 1987.

[2] D.G. Cahill, "Thermal conductivity measurement from 30 to $750 \mathrm{~K}$ : the $3 \omega$ method." Rev. Sci. Instrum., vol. 61 no. 2, pp. 802-808 1990.

[3] N.O. Birge, P.K. Dixon, and N. Menon, "Specific heat spectroscopy: Origins, status and applications of the $3 \omega$ method." Thermochim. Acta, 1997, vol. 304-305, pp. 51-66, 1997.

[4] C. Meffan, E. Calius, and V. Nock, "Thermal conductivity of PMMA-SiO2 phononic crystals measured by AC resistance thermometry', in Digest Tech. Papers IEEE Sensors Conference, Glasgow, UK, Oct 29-Nov 1, 2017.

[5] C. Clausen, T. Pedersen, and A. Bentien, "The 3Omega Method for the Measurement of Fouling Thickness, the Liquid Flow Rate, and Surface Contact." Sensors, vol. 17, no. 3, 552, 2017.

[6] C. Meffan and V. Nock, "AC resistance thermometry for thermal characterisation sensing inside a microfluidic channel." in Digest Tech. Papers ICONN'18 Conference, Wollongong, Australia, Jan 29- Feb 3, 2018.

[7] H. Natesan, and J. C. Bischof, "Multiscale Thermal Property Measurements for Biomedical Applications." ACS Biomater. Sci. Eng., vol. 3, no. 11, pp. 2669-2691, 2017.

[8] B.K. Park et al., "Thermal conductivity of biological cells at cellular level and correlation with disease state." J. Appl. Phys., vol. 119, no. 22, 224701, 2016.

[9] C. Dames, and Z. Chen, Applied Thermal Measurements at the Nanoscale: A Beginner's Guide to Electrothermal Methods, World Scientific Publishing 2018.

[10]Y. Sun et al., "A monolithic polydimethylsiloxane platform for zoospore capture, germination and single hypha force sensing." in Digest Tech. Papers Transducers Conference, Berlin, Germany, June 2327, 2019.

[11]B.H. Jo et al., "Three-dimensional micro-channel fabrication in polydimethylsiloxane (PDMS) elastomer." J. Microelectromech. S., vol. 9, no. 1, pp. 76-81, 2000.

[12] J. Bove et al., "Magnitude and Direction of Vesicle Dynamics in Growing Pollen Tubes Using Spatiotemporal Image Correlation Spectroscopy and Fluorescence Recovery after Photobleaching." Plant Physiol., vol. 147, no. 4, pp. 1646-1658, 2008

[13] V. Couvreur et al., "Going with the Flow: Multiscale Insights into the Composite Nature of Water Transport in Roots." Plant Physiol., vol. 178, no. 4, pp. 16891703, 2018.

[14] A. Muralidhar et al., "A pressure gradient facilitates mass flow in the oomycete Achlya bisexualis." Microbiology, vol. 162, no. 2, pp. 206-213, 2016.

\section{CONTACT}

*V. Nock, tel: +64-3-3694303;

volker.nock@canterbury.ac.nz 